\title{
PHOSPHORYLATION OF GLYCERIC ACID IN AQUEOUS SOLUTION USING TRIMETAPHOSPHATE
}

VERA KOLB* and LESLIE E. ORGEL**

The Salk Institute for Biological Studies, Post Office Box 85800, San Diego, CA 92186-5800, U.S.A.

(Received 27 July 1995)

\begin{abstract}
The phosphorylation of glyceric acid is an interesting prebiotic reaction because it converts a simple, potentially prebiotic organic molecule into phosphate derivatives that are central to carbohydrate metabolism. We find that $0.05 \mathrm{M}$ glyceric acid in the presence of $0.5 \mathrm{M}$ trimetaphosphate in alkaline solution gives a mixture of 2- and 3-phosphoglyceric acids in combined yields of up to $40 \%$.
\end{abstract}

Abbreviations: P3!, trimetaphosphate; 2-P, 2-Phosphoglycerate; 3-P, 3-Phosphoglycerate; TSPS, 3-trimethylsilyl propionic acid, sodium salt; NMR, nuclear magnetic resonance.

\section{Introduction}

Glyceric acid is a major product formed photochemically under conditions thought to simulate comet formation (Briggs et al., 1992, and references cited therein). Furthermore, although glyceric acid has not been reported as a product of Urey-Miller experiments, it must surely be formed along with serine, probably in large amounts when the concentration of ammonia is low (Miller and Orgel, 1974). Glyceric acid, therefore, is a potentially prebiotic molecule. However, it is the glyceric acid phosphates rather than glyceric acid itself that are central to carbohydrate metabolism (Moran et al., 1994). To complete the prebiotic synthesis of the molecules that provide an entry-point into carbohydrate metabolism it is necessary, therefore, to demonstrate the phosphorylation of glyceric acid under prebiotic conditions.

The trimetaphosphate ion (P3!) is among the most plausibly prebiotic phosphorylating agents. It reacts with cis-glycols including nucleosides to yield monophosphates, and less rapidly with monohydric alcohols to yield triphosphate esters. The reaction with glycols is rapid at pHs greater than 10 , but proceeds slowly even at $\mathrm{pH} 8$ and $25^{\circ} \mathrm{C}$ (Etaix and Orgel, 1978 and the references cited therein). We chose, therefore, to study the phosphorylation of glyceric acid with P3!.

\section{Materials}

We purchased from Sigma Chemical Company: DL-Glyceric acid, hemicalcium salt, hydrate; $\mathrm{L}(-)$ glyceric acid, hemicalcium salt, hydrate; $\mathrm{D}(+) 2$-phosphoglyceric

- Permanent address: Department of Chemistry, University of Wisconsin-Parkside, Kenosha, WI 53141-2000, U.S.A.

** To whom correspondence should be addressed.

Origins of Life and Evolution of the Biosphere 26: 7-13, 1996.

(C) 1996 Kluwer Academic Publishers. Printed in the Netherlands. 


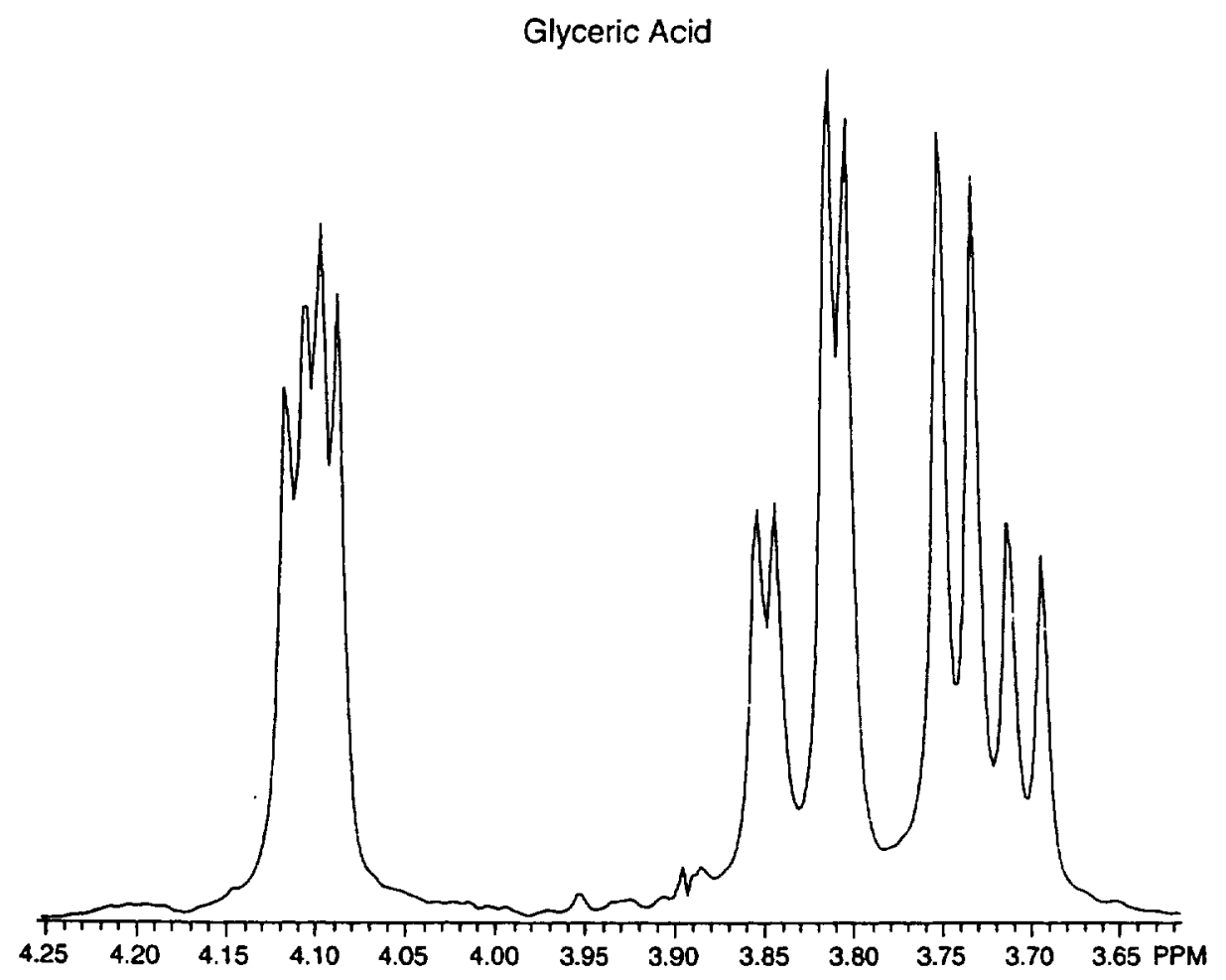

Fig. 1a. Proton NMR spectrum of glyceric acid.

acid, sodium salt, hydrate; and D(-)3-phosphoglyceric acid, disodium salt. Deuterated water; sodium deuteroxide, $40 \%$ and $30 \%$; deuterium chloride, $20 \%$; and 3-trimethylsilyl propionic acid, sodium salt (TSPS), were from Aldrich Chemical Company. Sodium trimetaphosphate, $\left(\mathrm{NaPO}_{3}\right)_{3} \cdot{ }_{3} \mathrm{H}_{2} \mathrm{O}$, was obtained from Johnson Matthey Electronics, and cation exchange resin, $A^{\circledR}$ 50W-X8, 50-100 mesh, hydrogen form, from Bio-Rad.

\section{Methods}

Sodium glycerate was prepared as follows. A column was packed with $15 \mathrm{~g}$ of cation exchange resin $A G^{\circledR} 50 \mathrm{~W}-\mathrm{X} 8$ suspended in water. The column was then washed with $0.1 \mathrm{M} \mathrm{NaOH}$ until the $\mathrm{pH}$ became basic, and then with water until the $\mathrm{pH}$ was neutral. A solution of $50 \mathrm{mg}$ of calcium glycerate in $250 \mathrm{ml}$ of water was applied to the column. The elute was collected and lyophilized, giving a virtually quantitative yield of sodium glycerate, as a hygroscopic white solid. Deuterated sodium trimetaphosphate was prepared by dissolving the hydrate in $\mathrm{D}_{2} \mathrm{O}$ and lyophilizing the resultant solution. 


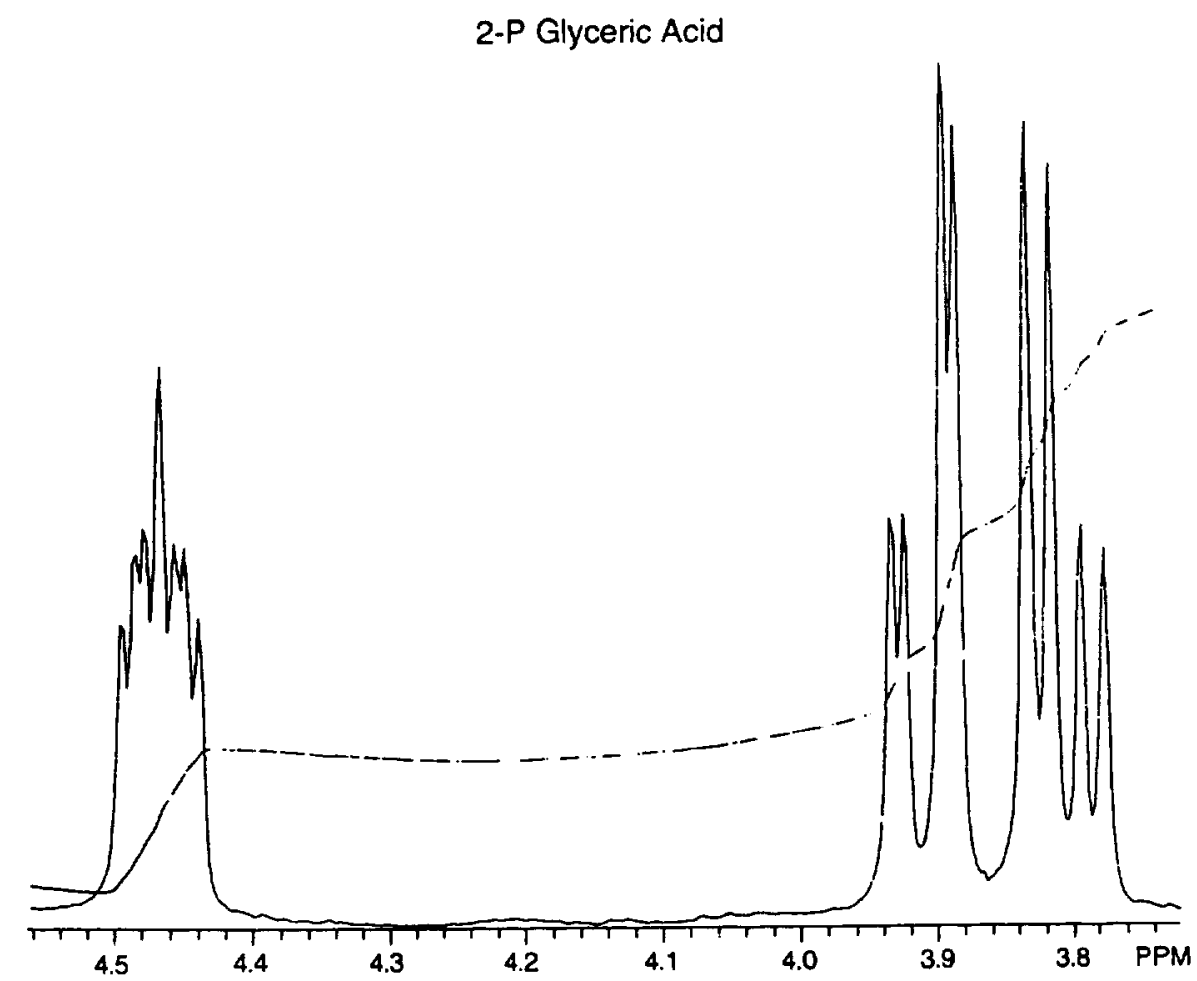

Fig. 1b. Proton NMR spectrum of 2-phosphoglyceric acid.

The reaction of sodium glycerate with $\mathrm{P} 3$ ! was studied in $\mathrm{D}_{2} \mathrm{O}$ solution to permit analysis of the products by ${ }^{1} \mathrm{H}$ NMR. In a typical experiment $360 \mathrm{mg}$ of deuterated sodium trimetaphosphate partially suspended in $\mathrm{D}_{2} \mathrm{O}(1 \mathrm{ml}$; equivalent to $1.0 \mathrm{M})$, and $0.1 \mathrm{M}$ sodium glycerate in $\mathrm{D}_{2} \mathrm{O}(1 \mathrm{ml})$ were mixed, and stirred magnetically. $\mathrm{NaOD}(30 \%)$ was added dropwise to the resulting solution as needed to maintain the $\mathrm{pH}$ within the range $10.5-11.9$. Samples of the solution $(800 \mu \mathrm{l})$ were taken for analysis at appropriate times.

Samples of the reaction mixture were brought to $\mathrm{pH} 8.5$ with $20 \% \mathrm{DCl}$ in $\mathrm{D}_{2} \mathrm{O}$, filtered, and introduced into the NMR tube, together with TSPS as an internal standard. NMR spectra were recorded on a GE QE-300 NMR with a 5-mm broad band and ${ }^{1} \mathrm{H},{ }^{13} \mathrm{C}$, or ${ }^{31} \mathrm{P}$ probes. Phosphoric acid was used as an internal standard for ${ }^{31} \mathrm{P}$ NMR spectroscopy.

\section{Results and Discussion}

We used ${ }^{1} \mathrm{H}$ NMR spectroscopy to study the phosphorylation of glyceric acid with $\mathrm{P} 3$ !. Fortunately the $\mathrm{CH}$ regions of glyceric acid (Figure $1 \mathrm{a}, 4.07-4.13 \mathrm{ppm}$ ), 2-P (Figure 1b, 4.42-4.52 ppm) and 3-P (Figure 1c, 4.15-4.23 ppm) do not overlap. 


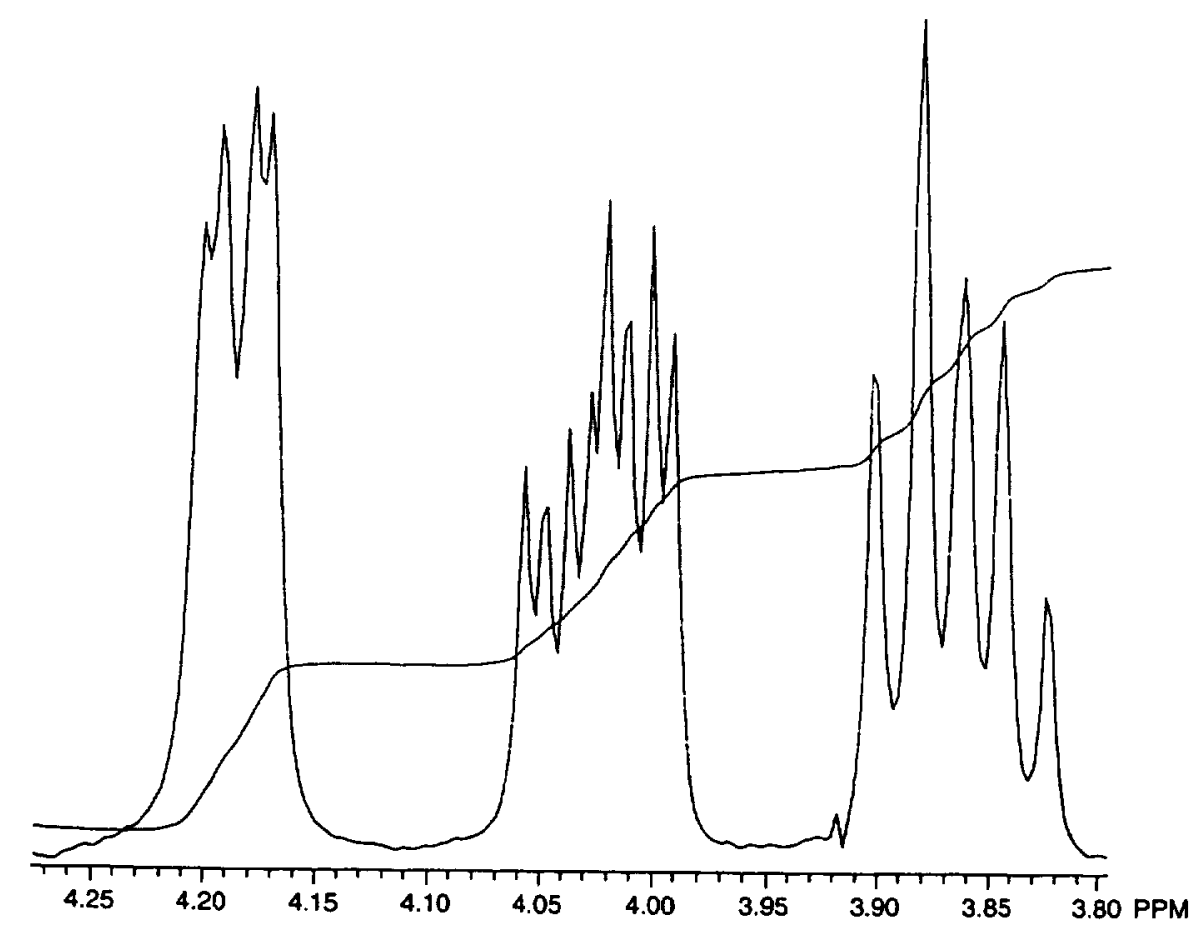

Fig. Ic. Proton NMR spectrum of 3-phosphoglyceric acid.

This permits the determination of the proportion of glyceric acid, 2-P and 3-P in a reaction mixture (Figure 1d).

After a number of preliminary experiments designed to determine the approximate half-time for the reaction (see above) we adopted the following near-optimal protocol. A solution containing $0.04 \mathrm{M}$ glyceric and $0.5 \mathrm{M}$ deuterated sodium trimetaphosphate in $\mathrm{D}_{2} \mathrm{O}$ was adjusted to $\mathrm{pH} 11.8$ with $30 \% \mathrm{NaOD}$. The $\mathrm{pH}$ was measured at short intervals, and the $\mathrm{pH}$ brought back to the range 11.2-11.6. About $37 \%$ of the theoretical requirement of $\mathrm{NaOD}$ was used up in the first 36 minutes, and a further $39 \%$ in the next three hours. The reaction mixture was then allowed to stand overnight. (A white precipitate formed, presumably of sodium tripolyphosphate). At this point a sample of the solution taken for NMR analysis contained about 17\% 2-P and 11\% 3-P. The bulk of the sample was then brought back to $\mathrm{pH}$ 11.7. The $\mathrm{pH}$ drifted back to $\mathrm{pH} 9.0$ in the course of $24 \mathrm{~h}$. NMR analysis then showed that the yields of 2-P and 3-P were about $21 \%$ and $17 \%$, respectively.

${ }^{31} \mathrm{P}$ NMR confirmed that $\mathrm{P} 3$ ! was extensively hydrolyzed to pyrophosphate and linear triphosphate in the course of the reaction. A small amount of orthophosphate was also formed. In addition 2-P and 3-P were identified (Figure 2). All peaks were identified by spiking the reaction mixture with authentic samples. The measured 


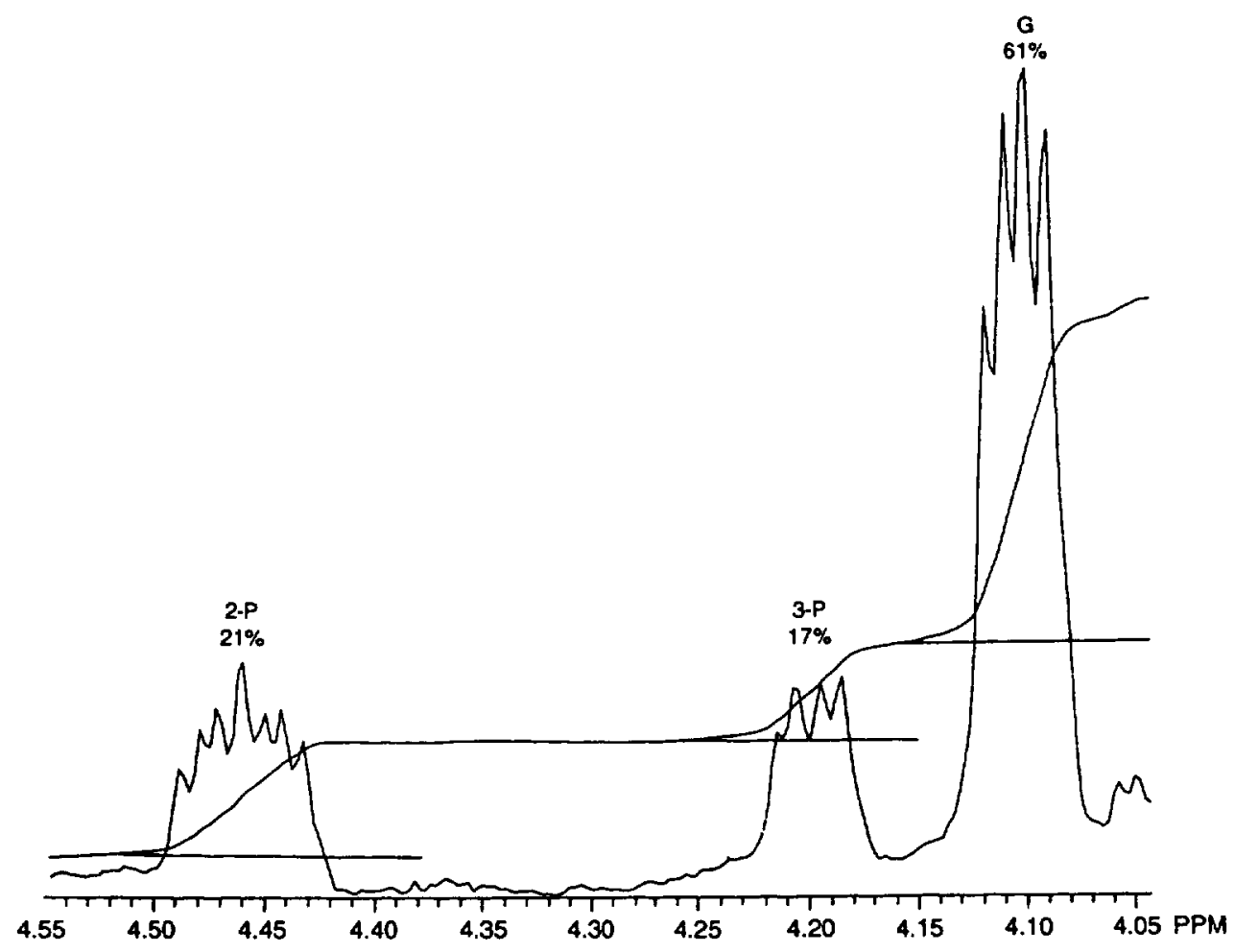

Fig. Id. Proton NMR spectrum of a typical product mixture from the phosphorylation of glyceric acid by sodium trimetaphosphate.

chemical shifts agreed with those previously reported (Gard et al., 1992; Gorenstein and Shah, 1984; Vogel, 1984).

The well-established mechanism for the phosphorylation of nucleosides by P3! enables us to suggest the scheme in Figure 3 for the phosphorylation of glyceric acid. We have not attempted to measure the rate of phosphorylation at neutral pHs, since our method of analysis does not detect small amounts of products. However, we can use the results of an analogous reaction, the phosphorylation of adenosine (Etaix and Orgel, 1978) to make a very rough estimate. The half-time for the phosphorylation of adenosine by $0.5 \mathrm{M} \mathrm{P} 3$ ! at pH 12 and $25^{\circ} \mathrm{C}$ is about $4 \mathrm{~h}$. The corresponding half-time at $\mathrm{pH} 8$ with $0.05 \mathrm{M}$ P3! is estimated to be somewhat in excess of 100 days. This suggests a rate reduction on going from $\mathrm{pH} 12$ to $\mathrm{pH} 8$ of roughly 60 . This estimate is very approximate since we do not take account of the changes in reagent concentrations that occur as the reaction proceeds. In our experiments, half of the final yield in the phosphorylation of glyceric acid at $\mathrm{pH}$ $\approx 11.5$ is obtained in less than $12 \mathrm{~h}$. Order of magnitude calculations based on these estimates suggest that significant phosphorylation of glyceric acid by $0.5 \mathrm{M}$ trimetaphosphate ion would occur at $\mathrm{pH} 8$ in a month. In the presence of $\mathrm{P} 3$ ! at 


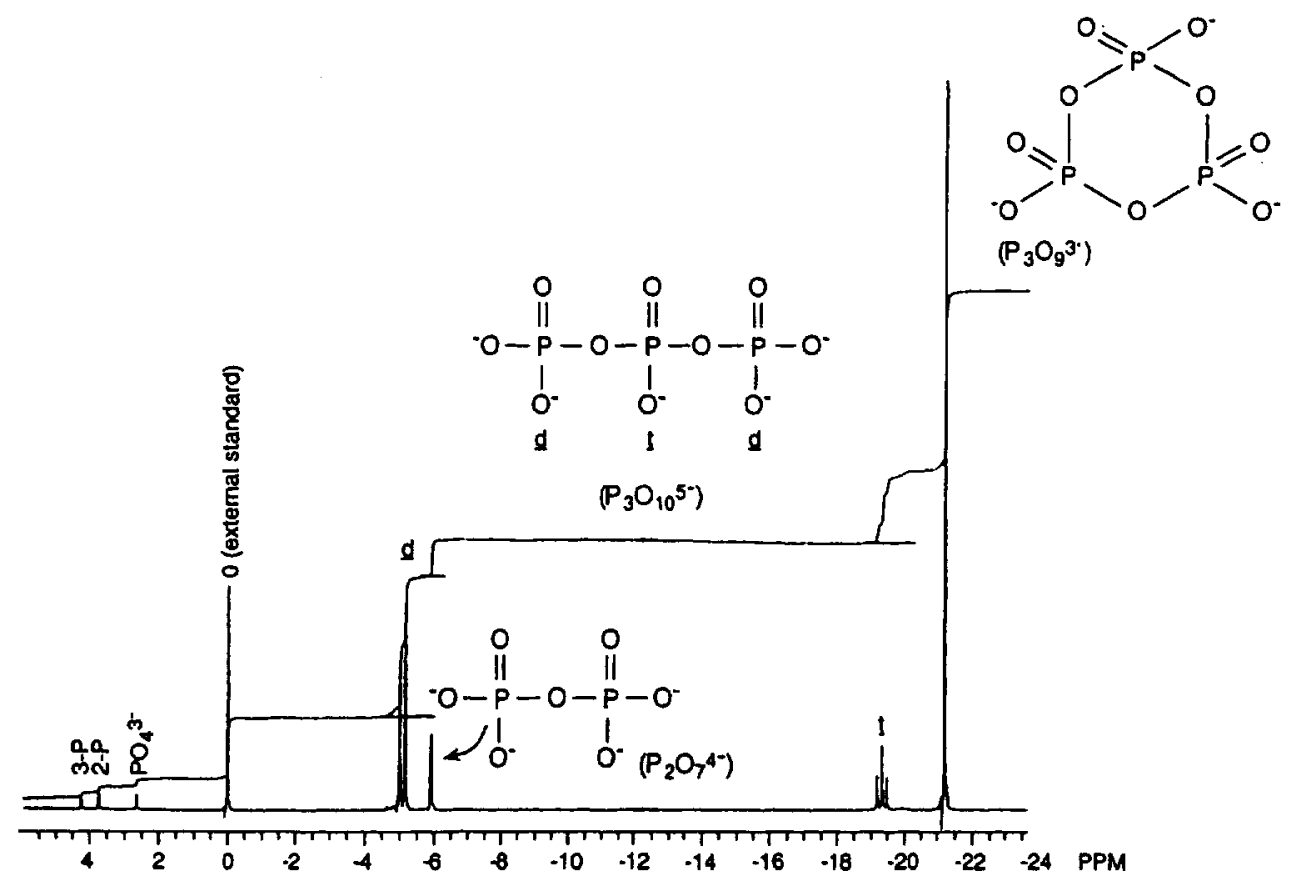

Fig. 2. Phosphorus NMR spectrum of a typical product mixture from the phosphorylation of glyceric acid by sodium trimetaphosphate.

a constant concentration of $1 \mathrm{mM}$ substantial phosphorylation might occur in 50 years.

Trimetaphosphate has been shown to form directly along with linear polyphosphates under simulated volcanic conditions (Yamagata et al., 1991). Under many conditions $\mathrm{P} 3$ ! is also the major product from the hydrolysis of long chain polyphosphates (Kalliney, 1972, and the references cited therein) compounds which are formed readily from ammonium hydrogen phosphate in the presence of urea (Osterberg and Orgel, 1972). Our synthesis of 2-P and 3-P from glyceric acid, therefore, can be considered a potentially prebiotic reaction.

\section{Acknowledgments}

This work was supported by NSCORT/EXOBIOLOGY Grant No. NAGW-2881 from the National Aeronautics and Space Administration. We thank Sylvia Bailey for manuscript preparation. 


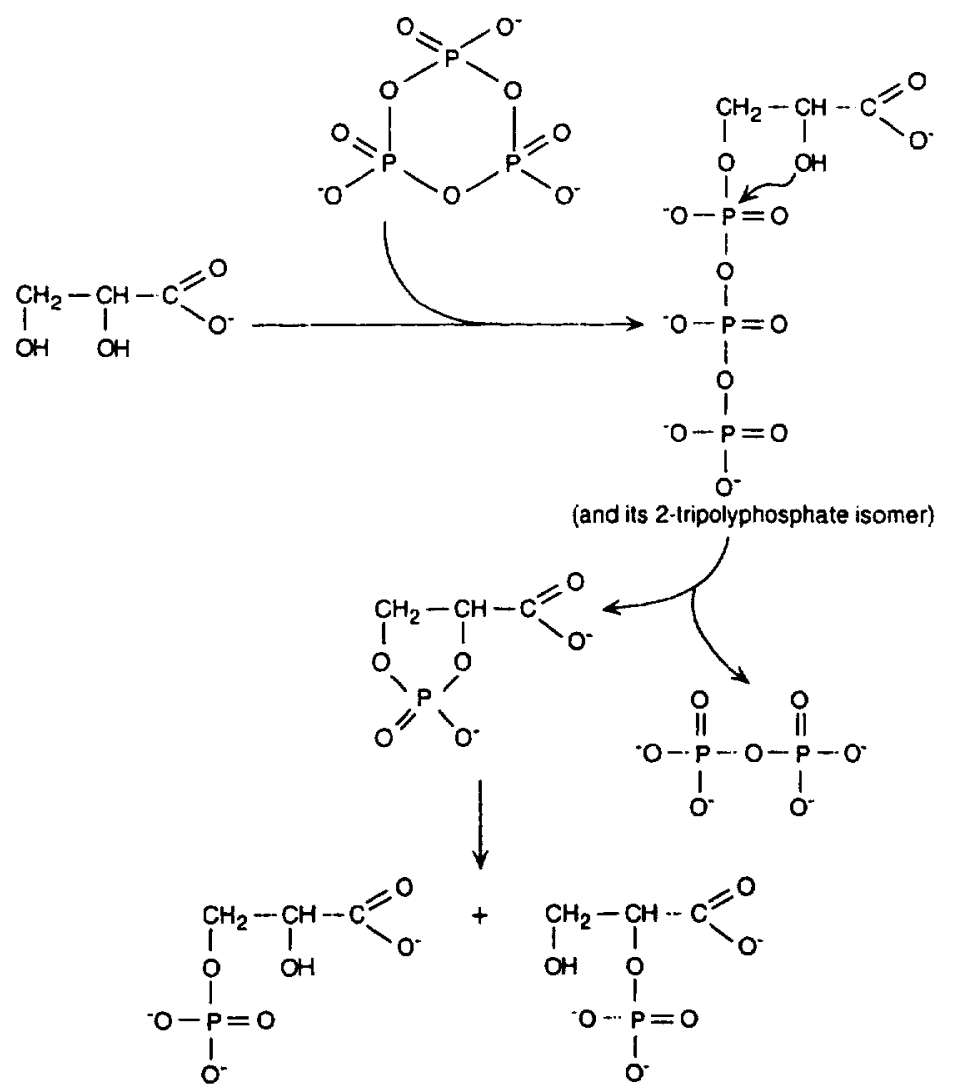

Fig. 3. The phosphorylation of glyceric acid by the trimetaphosphate ion.

\section{References}

Briggs, R., Ertem, G., Ferris, J. P., Greenberg, J. M., McCain, P. J., Menxosa-Gomez, C. X., and Schutte, W.: 1992, Origins of Life 22, 287-307

Etaix, E. and Orgel, L. E.: 1978, J. Carbohydrates-Nucleosides-Nucleotides 5, 91-110.

Gard, J. K., Gard, D. R. and Callis, C. F.: 1992, 'Quantitative analysis of inorganic phosphates using ${ }^{31}$ P NMR spectroscopy', pp. 41-55 in Phosphorus Chemistry. developments in American Science, American Chemical Society, Washington, D.C.

Gorenstein, D. G. and Shah, D. O.: 1984, 'Appendix III', pp. 572-573, in Phosphorus-31 NMR. Principles and Applications, Academic Press, Orlando, Florida.

Kalliney, S. Y.: 1972, 'Cyclophosphates', pp. 255-309 in Topics in Phosphorus Chemistry, Vol. 7. Interscience, New York.

Miller, S. L. and Orgel, L. E.: 1974, The origins of Life on the Earth, Prentice-Hall, Englewood Cliffs, New Jersey.

Moran, L. A., Scringenour, K. G., Horton, H. R., Ochs, R. S. and David, R. J., Ed.: 1994, Biochemistry, Prentice Hall, Englewood Cliffs, New Jersey.

Osterberg, R. and Orgel, L. E.: 1972, J. Mol. Evol. 1, 241-248.

Vogel, H. J.: 1984, '3'P-NMR studies of Phosphoproteins', pp. 105-154. In Phosphorus-31 NMR. Principles and Applications, Academic Press, Orlando, Florida.

Yamagata, Y., Watanabe, H., Saitoh, M. and Namba, T.: 1991, Nature 352, 516-519. 